\begin{tabular}{|c|c|}
\hline \multirow{3}{*}{ 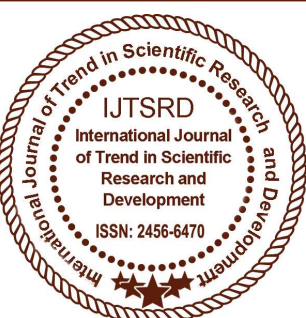 } & $\begin{array}{l}\text { International Journal of Trend in Scientific } \\
\text { Research and Development (IJTSRD) }\end{array}$ \\
\hline & International Open Access Journal \\
\hline & ISSN No: 2456 - 6470 | www.ijtsrd.com | Volume - 2 | Issue -3 \\
\hline
\end{tabular}

\title{
Lab scale production and optimization of PHB Biopolymer using orange peel by Pseudomonas spp. and Bacillus spp.
}

\author{
Mayur N. Rohi, Shivani Parwar, Ashok V. Gomashe \\ Department of Microbiology, S.S.E.S.A’s Science College, Congress Nagar, Nagpur, Maharashtra, India
}

\section{ABSTRACT}

Over the past 35 years, petrochemical derived plastics have become crucial materials in modern life. However, this oil based polymers take many years to degrade, which poses an environmental problem in some areas. An alternative for petrochemical plastic is biodegradable plastic, which is truly degradable, non- polluting and economically viable which can be degraded completely into natural compounds, such as $\mathrm{CO} 2$, methane, $\mathrm{H} 2 \mathrm{O}$ and biomass. Hence the objective of the current study was to produce ecofriendly and cost effective PHB from fruit waste i.e. Orange peels by Bacillus species and Pseudomonas species and to optimize the process at different parameters like Carbon source (15grams and 20grams) and Nitrogen source (4grams and 6grams). It was observed that Pseudomonas species showed the highest production of PHB. It was observed that by increasing the concentration of both Carbon and Nitrogen sources the production of PHB was strikingly enhanced where Bacillus species showed the highest production of PHB when grown under production media containing 20gms of Carbon and $6 \mathrm{gms}$ of Nitrogen sources. The presence of blueblack granules confirmed that the chosen organisms produced PHB.

Keywords: PHB, Bacillus, Pseudomonas, Orange Peels

\section{INTRODUCTION}

The conglomeration of petrochemical plastic waste in the environment is an increasing problem. In order to find alternative materials, researchers have developed fully biodegradable plastics, such as polyhydroxyalkanoates (PHAs). PHAs extracted from bacterial cells show materialistic properties that are similar to polypropylene (Braunegg et al. 1998). Many micro-organisms have the potential to degrade these macromolecules enzymatically (Mergaert et al. 1992). Over the past 35 years, petrochemical derived plastics have become crucial materials in modern life. However, this oil based polymers take many years to degrade, which poses an environmental problem in some areas. To overcome this problem, production of ecofriendly plastics (which can be decomposed by microorganisms) has been discovered. An alternative for petrochemical plastic is biodegradable plastic, which is truly degradable, nonpolluting and economically viable which can be degraded completely into natural compounds, such as $\mathrm{CO} 2$, methane, $\mathrm{H} 2 \mathrm{O}$ and biomass. This process is carried out by microorganisms. Other advantages of these materials over petrochemical plastics are that they are natural, renewable and biocompatible.

The occurrence of PHAs in bacteria has been known since 1920s, when Lemoigne reported the formation of poly 3-hydroxybutyrate (PHB) inside bacteria (Lemoigne, 1926). However the availability of lowcost petrochemical-derived plastics led to bioplastics being ignored for a long time due to its high production cost. Concern over petrochemical plastics in the environment has created a striking interest in biologically derived polymers. During recent years, intensive research has investigated the bacterial production of PHBs and a great effort is underway to improve this procedure (Braunegg et al. 2004; Khanna and Srivastava, 2005). Nonetheless, 
the PHA production price is still far above the price of conventional plastics (Salehizadeh and Van Loosdrecht, 2004).

PHB is produced by microorganisms (such as Ralstonia eutrophus, Methylobacterium rhodesianum or Bacillus megaterium) apparently in response to conditions of physiological stress (Ackermann et al. 1995) mainly in conditions where nutrients are limited. The polymer is primarily a product of carbon assimilation (from glucose or starch) and is employed by microorganisms as a form of energy storage molecule to be metabolized when other common energy sources are not available.

Most commercial plastics are synthetic polymers derived from petrochemicals. They tend to resist biodegradation. PHB-derived plastics are attractive because they are compostable and derived from renewables and are bio-degradable. Polyhydroxyalkanoates (PHAs), as one of these alternatives, have attracted much attention in recent years due to their varied mechanical properties, biocompatibility and biodegradability. They are synthesized and stored intracellularly by a wide variety of bacteria such as Bacillus sp., Pseudomonas sp., Azobacterium sp. etc.

Even though PHB is not water soluble, but it is $100 \%$ biodegradable in the environment when proper conditions such as sunlight, moisture, and oxygen are made available. Production of PHB and other bioplastics however are known to be very expensive since they are involving expensive carbon source. Apart from that, both the upstream processing and downstream processing also contributed to the high production cost. Therefore, studies have been actively conducted to find the suitable alternative to replace the original glucose. Hence the present study has been framed to analyze and utilize agricultural waste for production of PHB by Bacillus sp. and Pseudomonas $s p$.

\section{Materials and Methods}

\section{A. Culture Collection}

Bacillus sp. and Pseudomonas sp. were procured from the laboratory and the culture ere maintained at $4^{\circ} \mathrm{C}$ for further studies.

\section{B. Collection of Orange Peels}

Orange peels were collected from the fruit juice vendors situated at Nagpur region Maharashtra (India) and were taken to the laboratory in zip lock sterile polythene bags.

\section{Preparation of Extract}

Orange peels were grounded finely under sterile conditions, suspended in distilled water $(10 \mathrm{~g} / 100$ $\mathrm{ml}$ ) in 4 conical flasks each and then kept at room temperature for seven days. Then extract was filtered and the filtrate was sterilized at $110{ }^{\circ} \mathrm{C}$ for 10 minutes (Figure 1).

\section{Supplementation of Extract with Carbon and Nitrogen source}

Prior to inoculation, Carbon source was supplemented to the sterile filtrate to each flask. All the four flasks $(G)$ were supplemented with 10 grams of Glucose each and only two flasks were supplemented with 2 grams of amino acids lysine and histidine each additionally $(\mathrm{G}+\mathrm{N})$. Two flasks were inoculated with the cultures of Bacillus sp. (G, G+ N) and two with Pseudomonas sp. $(\mathrm{G}, \mathrm{G}+\mathrm{N})$ each. The inoculated flasks were then incubated at $37 \mathrm{C}$ for 7 days (Figure 2).

\section{E. Bacterial Staining}

After incubation, a loopful of culture was taken from the flasks on the slide and the cells were stained with Sudan black (5\%) and kept for 5 minutes. The slide was washed with tap water. The Slide was flooded with counter stain Safranine (5\%) and kept for 5 minutes. The slide was then washed off with tap water and the organisms were observed under microscope.

\section{F. Extraction and Purification of PHB}

The Filtrate was subjected to extraction using Centrifugation technique. The Filtrate was centrifuged at $4500 \mathrm{rpm}$ for 20 minutes. The supernatant was discarded and the pellet was stored separately at $4^{\circ} \mathrm{C}$. For purification the pellet was treated with equal quantity of sodium hypo chlorite and the mixture was centrifuged at 5000 rpm for 15 minutes. Then the pellet was washed 
with distilled water and acetone respectively. After washing the pellet was dissolved in $5 \mathrm{ml}$ of boiling chloroform and allowed to evaporate on sterile glass plate. After evaporation the dry powder was collected and weighed.

\section{G. Optimization}

The process of PHB production was optimized by considering different parameters like Carbon (15grams and 20grams) and Nitrogen (4grams and 6grams) sources at varied concentration. The procedure for optimizing the process of PHB production was similar as implemented above for its production.

\section{Result and Discussion}

The bioplastics have gained much attention in the recent scenario due to its ecofriendly effects. PHB is one of the components in the preparation of bioplastics. Bioplastics are preferred over petrochemical plastics as it can be degraded and does not create any polluting effects. Also the production of PHB is economic and serves ease of process modification, optimization and purification. Hence the objective of the current study was to produce ecofriendly and cost effective PHB from fruit waste i.e. Orange peels by Bacillus species and Pseudomonas species and to optimize the process at different parameters like Carbon source (15grams and 20 grams) and Nitrogen source (4grams and 6grams).

When the selected isolates of Bacillus and Pseudomonas species were allowed to grow in the production media containing Orange peels supplemented with both Carbon and Nitrogen sources showed the best production of PHB granules. Specifically Pseudomonas species showed the highest production of PHB (Table 1).

The process of PHB production was optimized by considering different parameters like Carbon (15grams and 20grams) and Nitrogen (4grams and 6grams) sources utilizing both Bacillus species and
Pseudomonas species. It was observed that by increasing the concentration of both Carbon and Nitrogen sources the production of PHB was strikingly enhanced where Bacillus species showed the highest production of PHB when grown under production media containing 20gms of Carbon and 6 gms of Nitrogen sources (Table 2, 3). From the staining technique, it was confirmed that PHB was produced by the bacterial cell (Figure 5).

In the current study, orange peel was used as the main substrate. The media was prepared and PHB production was carried out. The flasks were checked for the production of PHB by staining the mass with Sudan black (Figure 3,4). The presence of blue- black granules confirmed that the chosen organisms produced PHB. The PHB was then extracted and quantified. The similar results were reported by Sreeju et al. (2011); Sukan et al. (2014).

\section{Conclusion}

In this study, orange peel was used as a sole carbon source in the medium optimized for (PHB) production. These results clearly show that orange peel which is an abundant and unutilised agroindustrial waste material; it is a very attractive and an economically feasible carbon source for the production of PHAs. In the current study we have reported the production of (PHB) using orange peel as a sole carbon source with a very simple pre-treatment method. Also by optimizing the production process we can conclude that by increasing the concentrations of carbon and nitrogen sources in combination, the PHB productivity increases.

Although the strain selected was not a high PHB producer, the results obtained indicated that the presence of orange peel as a medium component promoted (PHB) production significantly. Further research is needed in downstream processing and further industrial strain selection in order to make this process more feasible for commercial production. 
International Journal of Trend in Scientific Research and Development (IJTSRD) ISSN: 2456-6470

TABLE I. PRODUCTION OF PHB BY PSEUDOMONAS AND BACILLUS SPECIES USING ORANGE PEELS

\begin{tabular}{|l|l|l|l|l|}
\hline Sr. No. & Name of Organism & $\begin{array}{l}\text { Concentration of } \\
\text { glucose }(\mathrm{G}) \text { source }\end{array}$ & $\begin{array}{l}\text { Concentration of glucose } \\
(\mathrm{G})+\text { nitrogen }(\mathrm{N}) \text { source }\end{array}$ & $\begin{array}{l}\text { Productivity } \\
(\mathrm{g} / 100 \mathrm{ml})\end{array}$ \\
\hline Flask 1. & $\begin{array}{l}\text { Pseudomonas } \\
\text { spp. }\end{array}$ & $10 \mathrm{~g}$ & - & $1.35 \mathrm{~g}$ \\
\hline Flask 2. & Pseudomonas spp. & - & $10 \mathrm{~g}+2 \mathrm{~g}$ & $1.40 \mathrm{~g}$ \\
\hline Flask 3. & Bacillus spp. & $10 \mathrm{~g}$ & - & $0.96 \mathrm{~g}$ \\
\hline Flask 4 & Bacillus spp. & - & $10+2 \mathrm{~g}$ & $1.12 \mathrm{~g}$ \\
\hline
\end{tabular}

TABLE II.OPTIMIZATION OF PHB PRODUCTION BY PSEUDOMONAS SPECIES IN ORANGE PEEL AT DIFFERENT GLUCOSE AND NITROGEN SOURCE COMBINATION

\begin{tabular}{|l|l|l|}
\hline Sr. NO. & Concentration of glucose $(\mathrm{G})+$ nitrogen source $(\mathbf{N})$ in $\mathrm{g}$ & Productivity (mg) \\
\hline $\mathbf{1 .}$ & $15 \mathrm{~g}+4 \mathrm{~g}$ & $1.87 \mathrm{~g}$ \\
\hline $\mathbf{2}$ & $20 \mathrm{~g}+6 \mathrm{~g}$ & $2.05 \mathrm{~g}$ \\
\hline
\end{tabular}

TABLE III.OPTIMIZATION OF PHB PRODUCTION BY BACILLUS SPECIES IN ORANGE PEEL AT DIFFERENT GLUCOSE AND NITROGEN SOURCE COMBINATION

\begin{tabular}{|l|l|l|}
\hline Sr. NO. & Concentration of glucose $(\mathrm{G})+$ nitrogen source $(\mathrm{N})$ in $\mathrm{g}$ & Productivity $(\mathrm{g})$ \\
\hline $\mathbf{1 .}$ & $15 \mathrm{~g}+4 \mathrm{~g}$ & $1.48 \mathrm{~g}$ \\
\hline $\mathbf{2}$ & $20 \mathrm{~g}+6 \mathrm{~g}$ & $2.34 \mathrm{~g}$ \\
\hline
\end{tabular}

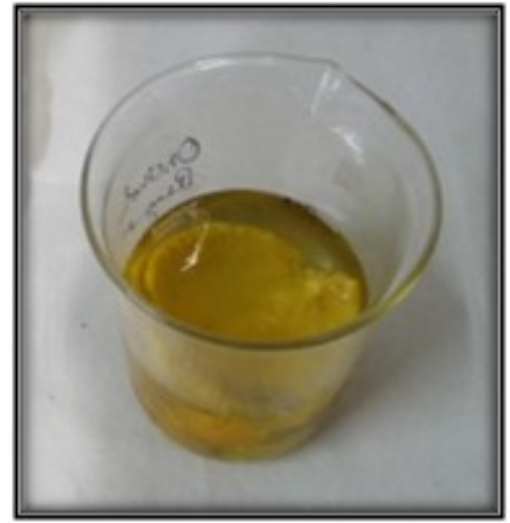

Fig.1 Orange peels suspended in distilled water

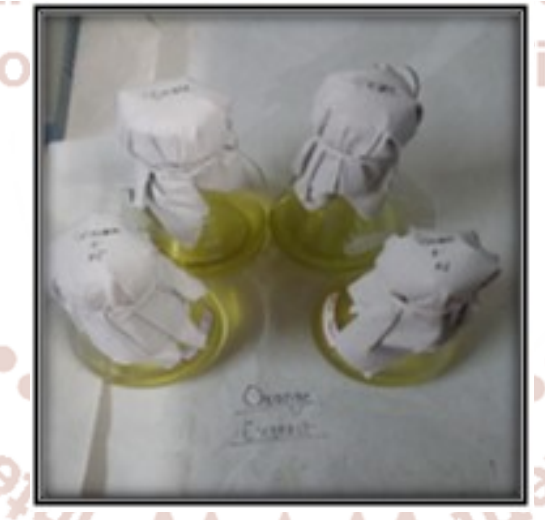

Fig.2 Orange extract with added carbon and nitrogen source

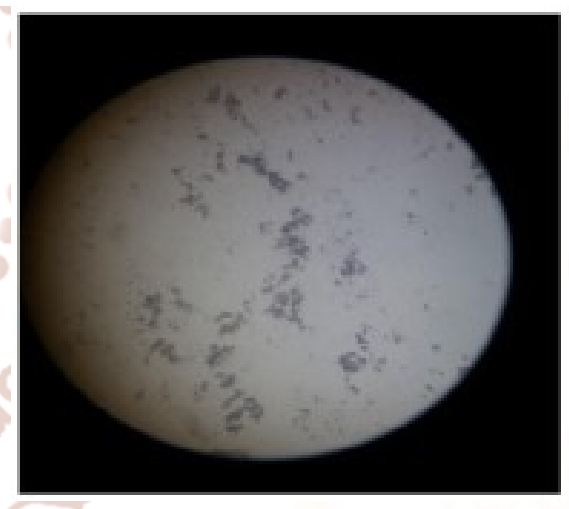

Fig.3 Bacillus species staining with Sudan black

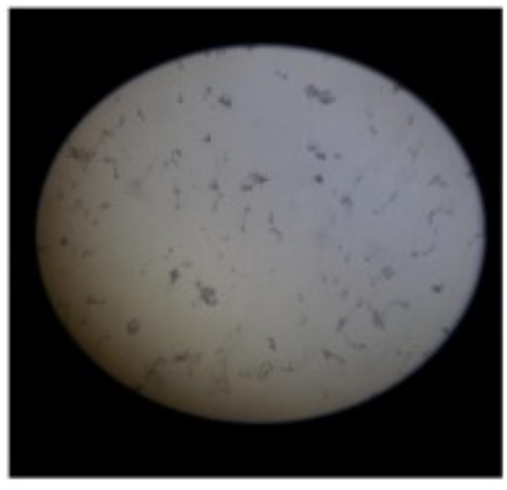

Fig.4 Pseudomonas species staining wit Sudan black

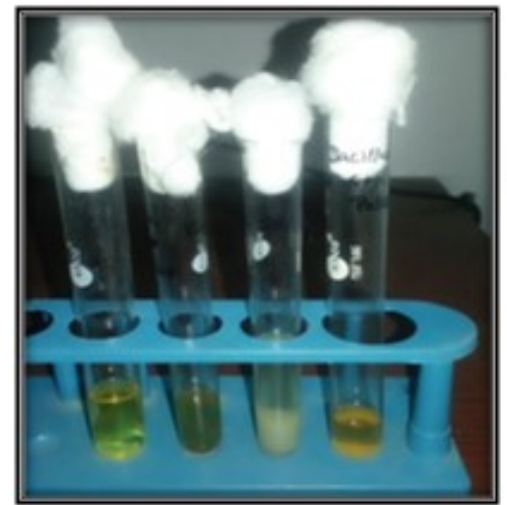

Fig.5: Extracted PHB after centrifugation 


\section{References}

1) Ackermann, Jörg-uwe; Müller, Susann; Lösche, Andreas; Bley, Thomas; Babel, Wolfgang (1995). "Methylobacterium rhodesianum cells tend to double the DNA content under growth limitations and accumulate PHB". Journal of Biotechnology. 39 (1): 9-20. doi:10.1016/01681656(94)00138-3

2) Acquel, Nicolas; Lo, Chi-Wei; Wei, Yu-Hong; Wu, Ho-Shing; Wang, Shaw S. (2008). "Isolation and purification of bacterial poly(3hydroxyalkanoates)". Biochemical Engineering Journal. 39 (1):

$15-$

27. doi:10.1016/j.bej.2007.11.029)

3) Braunegg, G., Bona, R. and Koller, M. (2004). Sustainable polymer production. Polym-Plast Technol 43, 1779-1793.

4) Braunegg, G., Lefebvre, G. and Genser, K.F. (1998). Polyhydroxyalkanoates, biopolyesters from renewable resources: Physiological and engineering aspects. $J$ Biotechnol 65, 127-161

Encyclopedia of Industrial Chemistry. doi:10.1002/14356007.n05_n07. ISBN 978-3-527-30673-2

8) Mergaert, J., Anderson, C., Wouters, A., Swings, J. and Kersters, K. (1992). Biodegradation of polyhydroxyalkanoates. FEMS

Microbiol Lett 103, 317-321.

9) Salehizadeh, H. and Van Loosdrecht, M.C.M. (2004). Production of polyhydroxyalkanoates by mixed culture: recent trends and biotechnological importance. Biotechnol Adv 22, 261-279.

10) Sreejus S.n. m.michael babu and c.mariappan, ( 2011). comparative study of biopolymer phb production from different Carbon sources and fruit peel wastes under different ph and time intervals for higher production journal of basic and applied science, issn 0973 - 8207

11) Steinbüchel, Alexander (2002). Biopolymers, 10 Volumes with Index. Wiley-VCH. ISBN 3-52730290-5.

12) Sukan, A., Roy, I. and Keshavarz, T. (2014).

5) Khanna,

S. and Srivastava, A.K. (2005a). Statistical media optimization studies for growth and PHB production by Ralstonia

eutropha. Process

Biochem 40, 2173-2182.

6) Lemoigne, M. (1926) Produits de dehydration et de polymerisation de l'acide $\beta$-oxobutyrique. Bull Soc Chim Biol 8, 770-782.

7) Lichtenthaler, Frieder W. (2010). "Carbohydrates Agro-Industrial Waste Materials as Substrates for the Production of Poly(3-Hydroxybutyric Acid). Journal of Biomaterials and Nanobiotechnology. 2014, 5, 229-240

13) Valappil, S. P., Rai, R., Bucke, C. and Roy, I. (2008). Polyhydroxyalkanoate Biosynthesis in Bacillus cereus SPV under Varied Limiting Conditions and an Insight into the Biosynthetic Genes Involved. Journal of Applied Microbiology. as Organic Raw Materials". Ullmann's 